Technical Report 1228

\title{
Leadership: Enhancing Team Adaptability in Dynamic Settings
}

\author{
Katherine J. Klein \\ University of Pennsylvania \\ Steve W. J. Kozlowski \\ Michigan State University
}

Basic Research Unit

Paul A. Gade, Chief

U.S. Army Research Institute for the Behavioral and Social Sciences 2511 Jefferson Davis Highway, Arlington, Virginia 22202-3926

April 2008 